\title{
Participación política de las mujeres en el Ecuador: elecciones seccionales 2014
}

\section{Political Participation of Women in Ecuador: The 2014 Subnational Elections}

\author{
Roxana Silva Ch.
}

Recepción: 23 de julio de 2014 Aceptación: 30 de agosto de 2014

\section{Resumen}

La participación política de las mujeres en América Latina se ha constituido un referente a nivel mundial; no obstante, todavía queda camino por recorrer para llegar a la ansiada participación equitativa e igualitaria. Ante esto, las mujeres latinoamericanas han fundando su lucha social en la reivindicación de la igualdad de derechos, pero también en la igualdad de oportunidades. El presente artículo hace un análisis sobre la situación actual de la participación de la mujer en América Latina, con especial énfasis de la participación en el Ecuador. El artículo considera la normativa vigente, la participación político electoral, los factores del sistema electoral que podrían afectar esta participación, y finalmente, se revisan las iniciativas que ha impulsado el Consejo Nacional Electoral del Ecuador en pro de la participación política de la mujer.

Palabras clave: participación, mujer, inclusión, Ecuador, proceso electoral.

\begin{abstract}
Political participation of women in Latin America has become a global benchmark, yet, there still some way to go in order to reach the desired equitable and equal participation. Given this, Latin American women have founded their social struggle by claiming equal rights but also equal opportunities. This article analyzes the current situation of women's participation in Latin America, with special emphasis on Ecuador. The article continues revising the Ecuadorian electoral law, the electoral participation of women, the electoral system factors that could affect such participation, and finally, it presents the initiatives promoted by the National Electoral Council of Ecuador in favor of women's participation.
\end{abstract}

Keywords: participation, women, inclusion, Ecuador, electoral process. 


\section{Introducción}

T as mujeres alrededor del mundo, han librado una dura lucha para asegurar que su voz sea escuchada y que sus intereses sean representados en la esfera pública, traduciéndose inmediatamente en innumerables esfuerzos por lograr una participación política equitativa y efectiva. América Latina, no ha sido ajena a esta realidad, siendo una región emergente en la que las mujeres han tomado roles protagónicos como figuras públicas y convirtiéndose rápidamente en un referente mundial en cuanto a la participación política de las mujeres. Sin embargo, en países como Ecuador aún falta un largo camino por recorrer y llegar a la ansiada participación equitativa e igualitaria, tanto en aspectos de la vida cotidiana, como en aspectos públicos, de los cuales la política es un eje fundamental.

La región latinoamericana se encuentra en un constante desarrollo. La adopción de modelos propios, basados en las vivencias y experiencias históricas de cada país, ha llevado a la redefinición de las estructuras sociales. No obstante, la desigualdad en diversos ámbitos, lamentablemente, persiste. La realidad de las mujeres ante estas dinámicas locales se ha visto afectada, llegando incluso a verse reflejada en una situación de injusticia social. Por un lado está la injusticia socioeconómica, que incluye la privación de bienes materiales para una vida digna, y por otro lado está la injusticia cultural o simbólica, que incluye la dominación cultural, el no reconocimiento y el irrespeto a las mujeres latinoamericanas (Fraser, 1997).

Es por esto, que en América Latina, los diversos colectivos de mujeres se han enfocado en exigir igualdad en las condiciones de vida, tanto públicas como privadas, para todas las personas, solo por el hecho de serlo. Es por esto, y de acuerdo al pensamiento de Elizabeth Stanton en el siglo XIX, "existen ciertos derechos naturales tan inalienables para la civilización como el derecho al aire (...) Los derechos naturales de un hombre y una mujer civilizados con el [derecho] al gobierno, a la propiedad, al desarrollo armonioso de todos sus poderes y a la gratificación 
de sus deseos. Los sexos son iguales y por lo tanto merecen derechos iguales" (Stanton, 1898).

Las mujeres latinoamericanas han fundando la lucha social en la reivindicación de la igualdad de derechos, pero también en la igualdad de oportunidades. Por lo tanto, y desde todos los países de la región, se ha impulsado el discurso de la igualdad en todas las esferas sociales y de intervención de las y los ciudadanos. Una igualdad que debe verse reflejada en el acceso a las mismas oportunidades para todas y todos los ciudadanos, sin distinción de género, etnia, religión, entre otros aspectos socioculturales que nos distinguen a unos de otros. Sin embargo, esta igualdad se ve debatida, el momento en que debiera tenerse en cuenta que debe existir una igualdad de partida; es decir, las mismas condiciones iniciales específicas de cada quien (León y Holguín, 2005).

La inclusión es sin duda un término amplio que se aplica a todos y cada uno de los ámbitos de la vida de las personas; sin embargo, la inclusión en los procesos de participación política se ha vuelto un eje fundamental de la lucha por el ejercicio pleno y democrático de los derechos de las mujeres, así como también para otros grupos que se conocen como grupos de atención prioritaria. Exigir oportunidades para participar, opinar y decidir en igualdad de condiciones sobre el futuro político de cada uno de nuestros países se ha vuelto un imperativo en el discurso de los colectivos de mujeres, presentando alternativas y opciones para lograr este cometido.

En la actualidad, la visibilización de otras y otros actores sociales que exigen equidad de derechos y de participación, ha reforzado las reivindicaciones que los colectivos de mujeres han impulsado desde finales del siglo XX en América Latina. Ante esto, los países han reaccionado de diferentes formas, siendo un claro ejemplo, la implementación de leyes y sistemas de cuotas para la representación, tanto de mujeres como de otras minorías. Sin embargo, indicar qué paises son más o menos inclusivo es complejo, pero ciertamente podemos analizar qué grupos tienen el derecho a participar en política. 
Con respecto a la situación política de las mujeres y su participación, es posible evidenciar que el grado de inclusividad de un régimen aumenta cuando ellas obtienen el derecho a sufragar y el derecho a ser elegidas, y hacen uso de estos dos; es decir, cuando sufragan y logran ingresar a las élites políticas, y hacen presencia en cargos públicos tanto de designación como de elección (Wills, 2004).

La inclusión política en los países latinoamericanos se ve entonces reflejada en distintos ámbitos de la vida política en cada uno de ellos, mediante los siguientes factores: el número de electoras que tienen la posibilidad de sufragar; el número de mujeres que en efecto ejercieron su derecho al sufragio; el número de mujeres que se presentaron como candidatas; el número de mujeres que resultaron electas como autoridades; y, el número de mujeres que son designadas para cargos públicos de alto rango, como ministras, secretarias de Estado, juezas, intendentas, entre otras designaciones.

En el análisis de la participación política de las mujeres en el Ecuador, se ha incluido una variable importante entre estos factores, variable que ayudará a determinar cuán inclusivo es en la práctica, su gobierno; esto es el número de mujeres que efectivamente participan en los espacios de toma de decisiones dentro de una organización política. Sumando todos y cada uno de estos aspectos, podemos apreciar una radiografía bastante acertada de la participación política real de las mujeres en cada uno de los países de América Latina, y del mundo también.

\section{Breve análisis del camino hacia la participación política de las mujeres en América Latina}

América Latina ha sentado precedentes en cuanto a la participación activa de la mujer en la esfera política. Esta participación activa en los asuntos públicos se ha dado como consecuencia positiva de una ardua lucha por exigir la participación justa y equitativa de las mujeres en los espacios de poder, así como de toma de decisiones de nuestros países. Las mujeres latinoamericanas han ganado espacios para potenciar la 
participación política, pero también han sido perjudicadas por los sistemas políticos patriarcales que hasta el día de hoy se mantienen vigentes.

Esto ha llevado a que varias mujeres vean mermada su participación política y afectados sus derechos. Por ejemplo, Lidia Gueiler Tejada, accedió en 1979 a la presidencia de Bolivia desde la presidencia de la Cámara de Diputados: ocho meses después sufrió un golpe de Estado y fue obligada al exilio. De la misma forma, Rosalía Arteaga, en febrero de 1997, ejerció la presidencia del Ecuador por tan solo 48 horas. Esto ocurrió tras el derrocamiento del presidente electo, Abdalá Bucaram, de quien era su vicepresidenta. En este caso, las estructuras políticas evidenciaron un machismo sistemático que impidió su designación como Jefa de Estado, principalmente por el hecho de ser mujer. Es así que, el entonces presidente del Congreso Nacional, Fabián Alarcón Rivera, asumió la presidencia interina hasta la celebración de nuevas elecciones en 1998.

Hoy en día, y con la segunda posesión presidencial de Michelle Bachelet en Chile, América Latina y el Caribe cuenta con seis mujeres gobernantes: Cristina Fernández en Argentina, Dilma Rousseff en Brasil, Laura Chinchilla en Costa Rica, Portia Simpson en Jamaica y Kamla Persad-Bissessar en y Trinidad y Tobago. Un dato curioso con respecto a este tema, es que Europa actualmente cuenta con la misma cantidad de mujeres en el poder: seis jefas de Estado o de gobierno en Alemania, Lituania, Dinamarca, Eslovenia, Kosovo y San Marino. Mientras que en África hay cuatro mujeres gobernantes en: Liberia, Malawi, República Centroafricana y Senegal. En Asia las mujeres gobiernan en Bangladesh, Corea del Sur y Tailandia.

Pese a que América Latina tiene un número significativo de mujeres como Presidentas o Jefas de Estado, la presencia en otros espacios de toma de decisiones en la esfera pública, todavía es escasa. Diversos estudios de organismos como la Organización de Naciones Unidas (ONU) o la Comisión Económica para América Latina y el Caribe (CEPAL) señalan una realidad latinoamericana, en la cual se refleja una participación bastante baja de las mujeres. En el continente las mujeres 
parlamentarias, juezas o ministras ocupan apenas un 26\% de los cargos; mientras que a nivel de los gobiernos locales la situación es todavía más precaria con un $11,7 \%$ de mujeres alcaldesas.

No obstante, América Latina continua llevando la posta para brindar oportunidades de participación a las mujeres. Actualmente, trece (13) países latinoamericanos tienen leyes de cuotas para que las mujeres participen activamente en política. Sin embargo, existen países en lo que se evidencian bajísima participación de las mujeres, como son Cuba y Nicaragua. Varias explicaciones pueden darse a este tema, sin embargo la piedra angular de la falta de participación femenina, es el trasfondo cultural, las tradiciones y costumbres de cada sociedad.

De hecho, las sociedades latinoamericanas tienen un tinte patriarcal, el cual define su estructura e invisibiliza, en la mayoría de los casos, los derechos de las mujeres. Sin embargo, con similar magnitud, factores socioeconómicos como la pobreza, educación, ubicación geográfica, entre otros, también afectan la posibilidad de que las mujeres se involucren en temas públicos, pues en muchos casos, están absortas en solucionar sus problemas personales, inmediatos y locales.

\section{La participación política de la mujer en el Ecuador}

En Ecuador, desde la aprobación de la Constitución en el 2008, se han establecido nuevos lineamientos, tanto de acción como de ejecución de las instituciones estatales. Dentro de estas nuevas directrices para la política pública nacional, está principalmente la inclusión como valor fundamental de la nueva institucionalidad ecuatoriana. Es por esto, que la inclusión no puede limitarse a lineamientos en el papel, sino que se tiene que llevarla a la práctica, brindar oportunidades y garantizar la participación equitativa de todos aquellos grupos o sectores sociales que han sido tradicionalmente relegados a la esfera privada, sin posibilidad de acción o decisión en lo público. Esta inclusión también se extiende a la arena política, la cual no solo se refiere a la participación a través del voto y de la postulación de todas y todos, sino también el derecho 
a estar presentes en igualdad de condiciones en los cargos de elección. En este sentido, la igualdad política también depende de si todos los grupos sociales del país están representados adecuadamente a nivel de gobierno (Htun, 2005).

Es por esto, que en Ecuador, a partir del marco normativo y legal vigente, se ha impulsado la inclusión a partir de políticas públicas y acciones afirmativas, generando oportunidades inéditas en la sociedad. En este sentido, las oportunidades, en efecto, se han extendido a la participación políticas de las y los ecuatorianos. Partiendo de la misma, la cual en su artículo 61 numeral 7, establece que las ecuatorianas y los ecuatorianos tienen el derecho de desempeñar empleos y funciones públicas con base en méritos y capacidades, y en un sistema de selección y designación transparente, incluyente, equitativo, pluralista y democrático, que garantice su participación, con criterios de equidad y paridad de género, igualdad de oportunidades para las personas con discapacidad y participación intergeneracional; se puede aseverar que se ha impulsado la participación política de las mujeres ecuatorianas, al menos desde la norma.

De la misma forma, en la Ley Orgánica Electoral y de Organizaciones Políticas, Código de la Democracia, se obliga que tanto las listas de candidaturas, como las directivas de las organizaciones políticas, sean paritarias y alternadas. Recogiendo lo establecido por Marta Lamas con respecto a la paridad, esta quiere decir estrictamente la mitad de mujeres y la mitad hombres (Lamas s/f). No obstante, la paridad va más allá de únicamente números, la paridad expresa un principio de igualdad, de no discriminación y de democratización (Arboleda, 2009).

Sin embargo, en el país todavía se adolece de un sistema electoral con debilidades, que repercuten negativamente en la representación paritaria de las mujeres en la práctica. El sistema electoral ecuatoriano se basa en la norma universal de un elector o electora es igual a un voto, partiendo de un principio de igualdad y equidad del voto de todas y todos los ciudadanos. Asimismo, la forma de votación es personalizada múltiple; es decir, las y los ciudadanos pueden votar por las y los candidatos de su preferencia entre distintas listas u organizaciones políticas. 
También pueden votar por una sola lista o en "plancha", que es lo que en Ecuador entendemos como el apoyo a todos y cada uno de los y las candidatas de una sola lista. Esta forma de votación, aporta a una distribución más personalizada del voto antes que reforzar el voto por una organización política. Sin embargo, en el Ecuador, más del 70\% del electorado vota por todos los candidatos de una sola lista.

Mientras que en lo que corresponde a la presentación de listas, el país tiene un sistema de listas abiertas o más conocido como sistema de listas libres, en las que de acuerdo a la votación ciudadana, la lista puede reordenarse. Por ejemplo, un candidato en el tercer puesto, puede ganar el segundo curul si recibe una votación mayor a la candidata que ocupa ese tercer puesto, lo que claramente perjudicaría la elección de dicha candidata. Sin embargo, según la Ley mencionada anteriormente, las listas de candidaturas deben ser alternadas, es decir, ser presentadas a través del conocido sistema de cremallera, así como paritariamente compuestas por el 50\% de hombres y el 50\% de mujeres. Esto no garantiza que tanto hombres como mujeres lleguen a ser autoridades de forma paritaria, pues en la gran mayoría de los casos, son hombres quienes encabezan las listas y quienes tienen mayores probabilidades de acceder a un escaño.

Otro factor que incide en la representación de las mujeres, son los métodos de asignación de escaños que se utiliza para todas las elecciones pluripersonales en el Ecuador. Para la elección de asambleístas provinciales, concejales, vocales de Juntas Parroquiales Rurales y parlamentarios andinos, rige la fórmula de divisores continuos, o mejor conocido como método D’Hondt; para la elección de asambleistas nacionales se aplica el método Webster. El primero de ellos es ampliamente conocido por su característica concentradora. De igual manera, otro factor que repercute en la participación y el acceso de la mujer a espacios de representación, es el tamaño de las circunscripciones electorales. Circunscripciones pequeñas junto con la fórmula de divisores continuos en el país, sin duda, ha dificultado la participación política de las mujeres; pues favorece a las organizaciones políticas mayoritarias, pero también reduce la representación de grupos minoritarios, dentro de los que podrían estar las mujeres. 


\section{Iniciativas inclusivas de la Función Electoral}

La Constitución del Ecuador marca un hito dentro de la protección a los grupos de atención prioritaria, pues convierte las políticas de inclusión en un eje transversal de la actuación de todas las funciones del Estado. En el caso de la Función Electoral, se ha impulsado la concreción de acciones afirmativas en la arena electoral. El Consejo Nacional Electoral creó la Comisión de Inclusión el 1 de noviembre de 2012, con el objetivo de generar insumos que permitan al organismo electoral adoptar las medidas necesarias en aras a garantizar la incorporación en igualdad de derechos a sectores tradicionalmente discriminados, y también con enfoque de género. Sin embargo a pesar de la normativa vigente en el país que obliga listas paritarias y alternadas, así como diversas iniciativas que impulsan la participación política de la mujer, deja un gran vacío, especialmente a nivel local, donde está bastante minimizada. Esta participación se ve tanto desde la perspectiva de las electoras, como de aquellas mujeres que participan en la competencia política como candidatas y resultan elegidas.

El sufragio en Ecuador, es un acto considerado como obligatorio; pues las personas reciben un certificado que valida todo trámite público o privado, por lo que esto dificulta medir con mayor veracidad la participación democrática tomando únicamente a la asistencia a las urnas el día de las elecciones como indicador. La votación de las ecuatorianas en los últimos 7 ańos no ha presentado mayor variación, mostrando un número superior de mujeres que acuden a votar en relación con los hombres. Sin embargo, esto está directamente relacionado con la cantidad de mujeres, que a su vez, también es superior a la de los hombres en el país. Pero una cifra importante e interesante, es que el número de mujeres que no acuden a votar ha ido disminuyendo conforme avanzan los procesos electorales. Este hecho sugiere un aumento de participación activa de las ecuatorianas como electoras. A pesar de que en el 2013 parecería haber un incremento, hay que tomar en cuenta que en ese ańo se incluyeron a los sectores con voto facultativo, como son las y los adolescentes, las personas con discapacidad y las y los adultos mayores, lo que incrementó el padrón y refleja un mayor 
ausentismo. Por el contrario, los hombres que no acuden a votar se mantienen en una cifra relativamente estable, y en el 2013 sucede lo mismo que en el caso de las mujeres (ver Tabla 1 y 2 ).

Tabla 1.

Evolución de la votación de las mujeres en el Ecuador $2006-2014$

\begin{tabular}{|c|c|c|c|c|}
\hline 2006 & 2009 & 2011 & 2013 & 2014 \\
\hline \multicolumn{5}{|c|}{ Mujeres empadronadas } \\
\hline 9165125 & 10532234 & 11158419 & 11675441 & 11618968 \\
\hline \multicolumn{5}{|c|}{ Electoras } \\
\hline 4623363 & 5275287 & 5588507 & 5848128 & 5821894 \\
\hline \multicolumn{5}{|c|}{ Total de sufragantes } \\
\hline 6617242 & 7928748 & 8634376 & 8602603 & 9600539 \\
\hline \multicolumn{5}{|c|}{ Sufragantes } \\
\hline 3399417 & 4052845 & 4400902 & 4373871 & 4909340 \\
\hline \multicolumn{5}{|c|}{ Mujeres que no sufragaron } \\
\hline 1223946 & 1222442 & 1187605 & 1474257 & 912554 \\
\hline
\end{tabular}

Fuente: Consejo Nacional Electoral, Dirección Nacional de Estadística Institucional, 2014

Tabla 2.

Evolución de la votación de los hombres en el Ecuador $2006-2014$

\begin{tabular}{c|c|c|c|c}
\hline 2006 & 2009 & 2011 & 2013 & 2014 \\
\hline \multicolumn{5}{c}{ Personas empadronadas } \\
\hline 9165125 & 10532234 & 11158419 & 11675441 & 11618968 \\
\hline 4541762 & 5256947 & 5569912 & 5827313 & 5797074 \\
\hline \multicolumn{5}{c}{ Total de sufragantes } \\
\hline 6617242 & 7928748 & 8634376 & 8602603 & 9600539 \\
\hline \multicolumn{5}{c}{ Sufragantes } \\
\hline 3217825 & 3875903 & 4233474 & 4228732 & 4691199 \\
\hline \multicolumn{5}{c}{ Hombres que no sufragaron } \\
\hline 1323937 & 1381044 & 1336438 & 1598581 & 1105875 \\
\hline
\end{tabular}

Fuente: Consejo Nacional Electoral, Dirección Nacional de Estadística Institucional, 2014 
Mientras que al analizar la participación política de las mujeres como autoridades electas, provenientes de un proceso de sufragio popular, existen dos escenarios para realizar el análisis: la Asamblea Nacional y los Gobiernos Autónomos Descentralizados (GAD). La participación política de la mujer en el espectro legislativo ha tenido una tendencia creciente en los últimos 15 ańos; sin embargo, aún queda camino por recorrer para alcanzar la paridad real en la representación.

Es importante tener en cuenta que, desde inicios del siglo XXI, se da un salto cuantitativo de más de 10 puntos porcentuales en la representación de las mujeres ecuatorianas en la legislatura, lo que representa un gran avance para la representación política de la mujer. No obstante, esto está directamente relacionado con la aprobación de la Ley de Cuotas aprobada en el año 2000. Esta Ley establecía un cupo mínimo de mujeres en las listas de candidaturas presentadas por las organizaciones políticas, así como exigía la ubicación alternada y secuencial de las y los candidatos en esta lista. Con la aprobación de esta Ley, se partió en el 2000 con una cuota del $30 \%$ de mujeres que deben conformar una lista, valor que incrementaría el $5 \%$ en cada proceso electoral. En el año 2008, con la aprobación del nuevo cuerpo constitucional, se estableció la paridad como norma en la presentación de candidaturas, llegando a una cuota del $50 \%$ para las mujeres y $50 \%$ para los hombres. Entonces, a partir del 2008, las listas fueron conformadas paritaria y alternadamente; sin embargo, no se puede asegurar una representación paritaria efectiva, pues esto depende del voto de los electores (ver Tabla 3). 
Tabla 3.

Representación de hombres y mujeres en el Legislativo 1998 - 2017

\begin{tabular}{c|c|c|c|c|c}
\hline Período Legislativo & $\begin{array}{c}\text { Total de } \\
\text { Legisladores }\end{array}$ & Mujeres & $\%$ & Hombres & $\%$ \\
\hline $1998-2003$ & 130 & 16 & $12,30 \%$ & 114 & $87,70 \%$ \\
\hline $2003-2007$ & 180 & 39 & $21,66 \%$ & 141 & $78,34 \%$ \\
\hline $2007-2011$ & 99 & 27 & $27,27 \%$ & 72 & $72,73 \%$ \\
\hline $\begin{array}{c}\text { Asamblea } \\
\text { Constituyente 2007 }\end{array}$ & 130 & 45 & $34,62 \%$ & 85 & $65,38 \%$ \\
\hline $2009-2013$ & 124 & 40 & $32,25 \%$ & 84 & $67,75 \%$ \\
\hline $2013-2017$ & 137 & 56 & $38,20 \%$ & 89 & $61,80 \%$ \\
\hline
\end{tabular}

Fuente: Consejo Nacional Electoral, Dirección Nacional de Estadística Institucional, 2014

En los GAD la realidad es diferente a aquella reflejada a nivel nacional en la Asamblea; lamentablemente la participación de las mujeres en los gobiernos locales en todo el país aún está muy minimizada. Las cifras hablan por sí solas, la participación local de las mujeres ecuatorianas no supera el 15\%; por lo que se puede aducir que en la cotidianidad de lo local, las mujeres siguen relegadas a roles propios de la esfera privada, con poca participación en la esfera pública o peor aún en la vida política de sus comunidades o localidades. En el terreno local, la cultura patriarcal se ve reforzada, con una mermada participación y exposición pública de las mujeres. 
Tabla 4.

Representación de hombres y mujeres en los GAD 2014 - 2019

\begin{tabular}{c|c|c|c|c|c}
\hline & Mujeres & \% Mujeres & Hombres & \% Hombres & Total \\
\hline Prefecturas & 2 & $8,7 \%$ & 21 & $91,30 \%$ & 23 \\
\hline Alcaldías & 16 & $7,3 \%$ & 205 & $92,7 \%$ & 221 \\
\hline Concejalías Urbanas & 294 & $33,7 \%$ & 573 & $66,3 \%$ & 867 \\
\hline Concejalías Rurales & 109 & $24,4 \%$ & 329 & $75,6 \%$ & 438 \\
\hline Juntas Parroquiales & 1023 & $24,8 \%$ & 3056 & $75,2 \%$ & 4079 \\
\hline Total & 1444 & $25,7 \%$ & 4184 & $74,3 \%$ & 5628 \\
\hline
\end{tabular}

Fuente: Consejo Nacional Electoral, Dirección Nacional de Estadística Institucional, 2014

En el Ecuador, para que las mujeres logren alcanzar cargos de elección popular correspondientes, por ejemplo, a prefecturas y alcaldías, es aparentemente un proceso complejo. Al constatar que de 23 prefecturas, apenas 2 tienen a mujeres como sus titulares, y que de las 221 alcaldías del país, tan solo 16 sean ocupadas por mujeres, resulta ser un hecho preocupante. Es así que, con estas cifras, la representación política de las mujeres difiere, abismalmente, a nivel nacional y local.

A pesar de que las organizaciones políticas han hecho un esfuerzo para que las listas pluripersonales cumplan con la Ley y puedan inscribir sus candidaturas, e incluso algunos partidos han tomado la decisión política de que algunas mujeres presidan las listas, lo que supone aumenta las posibilidades de ganar, en los resultados, no se refleja esta paridad, lo que está directamente vinculado con la forma de votación de las y los ciudadanos. Esto podría explicarse mediante la predominancia de una cultura política machista que se evidencia más al momento de elegir a autoridades locales. Así mismo, al ser la esfera local mucho más cercana, las y los votantes podrían preferir elegir a hombres que a mujeres de su entorno, debido a que existe la idea de que las mujeres debieran mantenerse en el espacio privado, mientras que los hombres están preparados para ejercer cargos públicos. En todas las sociedades se clasifica qué es 'lo propio' de las mujeres y 'lo propio' de los hombres, y desde esas ideas culturales se establecen las obligaciones sociales de 
cada sexo, con una serie de prohibiciones simbólicas (ver Lamas, 2007); quizás en las localidades ecuatorianas todavía 'lo propio' de las mujeres, no sea la política.

\section{Conclusión}

Sin duda alguna, la participación política de las mujeres en el Ecuador ha avanzado significativamente, pero es importante reconocer que aún queda un largo camino por recorrer. A pesar de la normativa vigente en el país obliga que se presenten listas paritarias y alternadas, así como diversas iniciativas que impulsan la participación política de la mujer, dicha participación, especialmente en los gobiernos locales en todo el país, aún se ve sumamente menguada. De igual manera, es innegable que las mujeres en los últimos años han asumido diversas posiciones de poder que eran tradicionalmente masculinas; sin embargo, la inequidad persiste en diversos campos, como el laboral, en los espacios de toma de decisión e incluso en la incursión en la política.

En suma, aún existen varias interrogantes: ¿son suficientes las acciones afirmativas o las leyes de cuotas? ¿O también es necesario pensar en acciones afirmativas de llegada, como cuotas o escaños obligatorios para mujeres, para asegurar la representación política de las mujeres tanto a nivel nacional como local? La participación política de las mujeres es un gran reto para la sociedad ecuatoriana, ahora somos actoras visibles, pero para permitir que estos cambios avancen, debemos continuar actuando con transparencia, justicia, equidad e inclusión de todos y todas. 


\section{Bibliografía}

Arboleda, M. (2009). "Mujeres hacia la paridad: cambios moleculares y resistencias al cambio" en Revista La Tendencia No9, Flacso Andes.

Fraser, N. (1997). Iustitia interrupta. Bogotá: Universidad de los Andes. Htun, M. (2005). "Democracia e inclusión política: La región andina en perspectiva comparada" en León M. (ed.) Nadando contra la corriente: Mujeres y cuotas politicas en los paises andinos,. Bogotá: Universidad Nacional, pp. $15-39$.

IFAD, International Fund for Agricultural Development. (2012). Gender equality and women's empowerment. Recuperado de http:// www.ifad.org/gender/index_full.htm. (Acceso 6 de noviembre de 2013).

Lamas, M. (2013). “Ciudadanía, Feminismo y Paridad” en PNUD América Latina Genera. Recuperado de http://www.americalatinagenera.org/es/index.php?option $=$ com_content \&task=view\&i$\mathrm{d}=905 \&$ pub_id=618 (Acceso 6 de noviembre de 2013).

León, M. y Holguín, J. (2005). "La cuota sola no basta: El caso de Colombia” en León M. (ed.). Nadando contra la corriente: Mujeres y cuotas politicas en los paises andinos, Bogotá: Universidad Nacional, pp. $41-89$.

Cárdenas Ońate, M. (2007). Mujeres en la historia del Ecuador, género y cultura. Quito: IPANC

Mena, C. (2007). "Aproximación al cruce entre la cultura y el género. Una mirada desde los derechos de las mujeres" en Mujeres en la historia del Ecuador, género y cultura. Quito: IPANC

Stanton, E. (1898). Eighty Years and More (1815-1897); Reminiscences of Elizabeth Cady Stanton. New York City: Humanity Books.

Wills, M. (2004). "Las trayectorias femeninas y feministas hacia lo público en Colombia (1970-2000). ¿Inclusión sin representación?” disertación presentada para obtener el título de Ph.D. en la Universidad de Austin, Texas, Agosto. 